\title{
La antropología y la sociología del Derecho como formación interdisciplinaria
}

\author{
ANTONIO PEÑA JUMPA*
}

SUMARIO: INTRODUCCIÓN.- I. ¿QUÉ ENTENDER POR «FORMACIÓN INTERDISCIPLINARIA»?- II. LA FORMACIÓN INTERDISCIPLINARIA COMO ANTROPOLOGÍA JURÍDICA.- III. FORMACIÓN INTERDISCIPLINARIA COMOSOCIOLOGÍA JURÍDICA.- IV. RETOS Y DESAFÍOS EN EL TRABAJO INTERDISCIPLINARIO.

\section{INTRODUCCIÓN}

Las páginas siguientes transcriben sintéticamente la exposición realizada en el seminario sobre Enseñanza del Derecho realizada en agosto de 2010 en la Facultad de Derecho de la Pontificia Universidad Católica del Perú. A partir de la experiencia en los cursos de Sociología del Derecho, Proyección Social del Derecho, Antropología del Derecho, Teoría General del Derecho e Investigación Jurídica, nos permitimos reflexionar acerca de la interrelación de dos macro disciplinas, la sociología y la antropología, con una tercera macro disciplina: el Derecho.

Pretendemos absolver las siguientes preguntas:

- ¿Qué entender por «formación interdisciplinaria»?

- ¿Cuál es nuestra experiencia de la interrelación de la antropología y el Derecho como formación interdisciplinaria?

- En el mismo sentido, icuál es nuestra experiencia en la interrelación de la sociología y el Derecho como formación interdisciplinaria?

- Por último, icuáles son los retos y desafíos sobre esta experiencia interdisciplinaria?

I. ¿QUÉ ENTENDERPOR «FORMACIÓN INTERDISCIPLINARIA»? Para responder la pregunta, nos permitimos distinguir entre «lo interdisciplinario» y «la formación interdisciplinaria». Antes de aproximarnos propiamente a «la formación interdisciplinaria», es importante absolver lo que entendemos por «lo disciplinario».

\section{I.1. Lo interdisciplinario}

Por «lo interdisciplinario» entendemos la interacción e integración de dos o más disciplinas para un mismo fin. De esta definición, el concepto 
de «interacción» consiste en la mutua correspondencia, en términos de aportes o cuestionamientos, de dos o más disciplinas. El concepto de «integración» se refiere a la posibilidad de trabajo conjunto, a pesar de sus diferencias, de dos o más disciplinas. Estos dos conceptos nos llevan a afirmar que, para que se produzca un trabajo interdisciplinario, es necesario que las disciplinas que «interactúan» y se «integran» no deben ser disímiles o, cuando menos, deben tener un nivel de comunicación.

Por ejemplo, cuando interactuamos e integramos la sociología y la antropología con el Derecho, encontramos que las tres tienen una comunicación común: estudian las relaciones humanas. La sociología estudia las relaciones sociales de un grupo social o de los seres humanos, la antropología estudia las relaciones culturales del mismo grupo social o de los seres humanos, mientras el Derecho estudia las relaciones normativas del mismo grupo social o de los seres humanos.

De otro lado, el concepto de «fin» en «lo interdisciplinario» tiene una multitud de posibilidades. Para los fines de nuestra experiencia, destacamos dos en las que pueden converger la sociología, la antropología y el Derecho:

- Conocer un fenómeno determinado o el trabajo de investigación

- Aprender y enseñar una determinada materia o tema, o el trabajo de educación

El acto de conocer un determinado fenómeno nos conecta con un proceso de comprensión y recolección de información que se puede sistematizar en la investigación. En tal posibilidad, las disciplinas como la sociología, la antropología y el Derecho interactúan y se integran dentro del propósito de investigar el fenómeno. El muto aporte y el trabajo conjunto se produce para conocerlo.

El acto de aprender y enseñar una determinada materia o tema se relaciona con un trabajo educativo. En este caso, las dos o tres disciplinas que interactúan han utilizado sus contenidos, métodos y técnicas - en interacción e integración - para facilitar la enseñanza de la materia o tema a tratar. Por ejemplo, el Derecho hace más referencia a normas, la sociología, a hechos y la antropología, a conductas para comprender o discutir el contenido de un tema determinado.

Sea el fin de investigación o el fin educativo, las disciplinas que intervienen lo hacen con la riqueza de medios que tiene cada una de ellas. El conocimiento, el lenguaje, los procesos o procedimientos, las estrategias, etcétera, de cada disciplina ayudan para dichos fines.

Nótese que el concepto de «lo interdisciplinario» es diferente a «lo multidisciplinario». En este último caso las disciplinas pueden interactuar, pero no están integradas. También, «lo interdisciplinario» es diferente a lo que, últimamente, se está denominando «lo transdisciplinario». 
En este existe integración, pero se busca ir más allá de lo que aportan las disciplinas, y se pierde la interacción. «Lo interdisciplinario» no tiene como propósito conformar una nueva disciplina —supra o infra-, sino el enriquecimiento permanente de las dos o más disciplinas que interactúan y, en tal sentido, se integran.

\subsection{La formación interdisciplinaria}

Definido «lo interdisciplinario», «la formación interdisciplinaria» puede entenderse como un conocimiento preestablecido, pero también como un efecto en el conocimiento que produce la interacción e integración de dos o más disciplinas. El conocimiento preestablecido tiene un símil con el aprendizaje, en tanto todo conocimiento tiene una causa u origen; es decir, se recibe o se coge de los hechos o de la interrelación de los propios seres humanos.

Por su parte, el efecto en el conocimiento tiene, a su vez, un símil con la enseñanza, en tanto todo conocimiento puede y debe transmitirse; esto es, se comparte para la propia comprensión de los hechos o para la propia interrelación de los seres humanos. Entonces, aprendizaje y enseñanza están muy presentes. $O$ son elementos fundamentales, en la formación interdisciplinaria.

Si tenemos en cuenta esta definición, pedemos encontrar la formación interdisciplinaria en, al menos, tres niveles, de los cuales dos coinciden con los fines anteriormente presentados para definir «lo interdisciplinario»:

- En el trabajo de investigación

- En el trabajo educativo

- En el ejercicio profesional

En el trabajo de investigación, la formación interdisciplinaria significa aproximarnos a la compresión de un determinado fenómeno con el aprendizaje y la enseñanza de las disciplinas que intervienen. En el trabajo educativo, la formación interdisciplinaria significa la aplicación del aprendizaje y la enseñanza de las disciplinas que intervienen para la transmisión y aprehensión del conocimiento de estas en un receptor determinado. Por último, en el ejercicio profesional, la formación interdisciplinaria significa la aplicación del aprendizaje y la enseñanza de las disciplinas que intervienen en la planificación y ejecución de proyectos o políticas, en la prestación de servicios, y en la resolución de problemas o casos.

Ahora que tenemos en cuenta esta breve teoría, pasamos a explicar su uso en la experiencia de la antropología y la sociología del Derecho. 


\section{LA FORMACIÓN INTERDISCIPLINARIA COMO ANTROPOLOGÍA JURÍDICA}

Por limitaciones académicas, la formación interdisciplinaria en la experiencia de la antropología jurídica solo la abordaremos a dos niveles: en el trabajo de investigación y en el trabajo educativo. Dejamos pendiente su tratamiento en el nivel del ejercicio profesional.

\section{II.1. Experiencia de la antropología jurídica en el trabajo de investigación}

Investigar a través del Derecho y la antropología supone el uso de conocimientos, métodos y técnicas diferentes, como se ha adelantado. Veamos cómo se aproxima la antropología jurídica, cuando partimos de un fenómeno determinado para la investigación.

Tomemos como ejemplo un caso de conflicto; de maltratos físicos entre los miembros de un matrimonio. El Derecho se aproxima a este fenómeno desde una concepción lógica compuesta por normas que regulan los hechos. Para empezar, se pregunta si el matrimonio es formal o de hecho, y, si lo fuere, inmediatamente relaciona las normas que regulan los derechos y obligaciones de la pareja, y establece las posibilidades de una causal de separación o divorcio, más allá de las responsabilidades civiles y penales que corresponden por las lesiones que se hubieren producido.

También, el Derecho puede incluir la aplicación de procedimientos flexibles para resolver el conflicto: si las lesiones no fueron graves y se puede recomponer la relación de pareja, se puede optar por una separación temporal y el tratamiento terapéutico del miembro del matrimonio que causó el maltrato. En ambas situaciones, el análisis de las normas vigentes y aplicables al caso constituye la técnica más importante que aporta el Derecho.

Por su parte, la antropología se aproxima desde un conocimiento, un método y una técnica diferentes. Esta ve el conflicto de los miembros del matrimonio bajo el conjunto de relaciones culturales que giran alrededor de este. Interesa conocer la formación e identidad cultural de los actores del conflicto, de sus respectivas familias, y, de ser posible, del grupo social al que pertenecen. Con herramientas como la observación, las entrevistas y los testimonios, se preguntará por las causas del conflicto más que por sus efectos, como lo haría el Derecho. Antes que buscar la solución del conflicto en los miembros del matrimonio, la antropología busca comprender el origen del problema, y recién importa la solución en segundo o tercer nivel.

Ahora, la interacción e integración de ambas disciplinas supone investigar el fenómeno estudiando sus causas antropológicas y sus efectos jurídicos. Conocimientos, métodos y técnicas de ambas disciplinas interactúan y se integran en el propósito de comprender el fenómeno y 
proyectar algunas soluciones. Si actuara solo una de las disciplinas, la aproximación al fenómeno tendría solo uno de los aportes.

\section{II.2. Experiencia de la antropología jurídica en el trabajo educativo}

LA ANTROPO-

LOGÍA Y LA

SOCIOLOGÍA DEL

DERECHO COMO

FORMACIÓN

INTER-

DISCIPLINARIA cando» - , se recurre al conocimiento, método y técnicas de ambas disciplinas. Esto es, de una parte, se recurre al conocimiento normativo de la actuación de las partes en conflicto (el Derecho) y, de otro lado, se recurre al conocimiento causal cultural de los actores del mismo conflicto y su contexto (la antropología).

Si utilizamos esta aproximación, el trabajo educativo desde la antropología del Derecho consistirá en:

- Transmitir el conocimiento del fenómeno o tema de estudio bajo un marco conceptual que incluya algo más que la ley y más que el propio contexto cultural.

- Comprender las conductas de los actores del conflicto bajo un contexto de pluralismo jurídico. Esto significa que existen reglas o normas entre las partes del conflicto que incluyen y, al mismo tiempo, confrontan el orden jurídico oficial y el orden social o cultural que los identifica.

- Promover el uso del trabajo de campo como método de estudio para el educando. Es decir, de forma complementaria al estudio doctrinario y teórico del Derecho, se exige la aplicación de técnicas de campo como la observación, las entrevistas y el registro de testimonios.

- Recurrir a una bibliografía académica de autores que han desarrollado ambas disciplinas en el tema de estudio. Por ejemplo, en el tema de los conflictos y la teoría de la antropología aplicada al Derecho es posible recurrir a Bronislaw Malinowski ${ }^{1}$, Leopold 


\section{Pospisil ${ }^{2}$, Sally Falk Moore 3 , Laura Nader ${ }^{4}$, John Griffiths ${ }^{5}$, Sally}

Engle Merry y y Marie Claire Foblets ${ }^{7}$, entre otros.

Solo la interacción e integración de los conceptos, métodos y técnicas del Derecho y la antropología permitiría una comprensión y perspectiva del tema, o problema, que se enseña en la dimensión descrita. El Derecho y la antropología por separado no conseguirían el mismo resultado.

\section{III.FORMACIÓN INTERDISCIPLINARIA COMO SOCIOLOGÍA JURÍDICA}

Si seguimos la misma división aplicada para la antropología del Derecho, en la sociología del Derecho también se puede distinguir entre trabajo de investigación y trabajo educativo. A continuación, nos referimos a ellos ampliando nuestra fuente de información teórica basada en un sociólogo del Derecho clásico: Max Weber ${ }^{8}$.

2 PosPISIL, Leopold. The ethnology of law. Menlo Park, California: Cummings Publishing Company, 1978; Anthropology of law, a comparative theory. Nueva York, Evanston, San Francisco y Londres: Harper y Row Publishers, 1971; "Structural change and primitive law: consequences of a Papuan legal case». En Laura Nader (editor). Law in culture and society. Chicago: Aldine Publishing Company, 1969; Kapauku Papuans and their law. New Haven: Yale University Publications, 1958.

3 MOORE, Sally Falk. Law and anthropology — a reader. Malden, Oxford, Victoria: Blackwell Publishing, 2005; Law as a process. An anthropological approach. Londres, Henley y Boston: Routledge \& Kegan Paul, 1978; «Law and social change: the semi-autonomous social field as an appropriate subject of study». Law and Society Review, vol. 7, № 4, 1973; «Legal liability and evolutionary interpretation: some aspects of strict ability, self-help and collective responsibility». En Max Gluckman (editor). The allocation of responsibility. Manchester: University Press, 1972; Power and property in Inca Peru. Nueva York: Columbia University Press, 1958.

4 NADER, Laura. "The influence of dispute resolution on globalization: the political economy of legal models». En Johannes Feest (editor). Globalization and legal cultures. IISL Oñati Paper $\mathrm{N}^{\circ}$ 7. Oñati, 1997; Harmony, ideology: justice and control in a Zapotec mountain village. California: Stanford University Press, 1990; Law in culture and society. Chicago: Aldine Publishing Company, 1969; «The Anthropological study of law». American Anthropologist, vol. 67, N 6, parte 2, diciembre de 1965, reimpreso en Peter Sack y Jonathan Aleck (editores). Law and anthropology. Aldershot, Hong Kong, Singapur y Sydney: Dartmouth, 1992.

5 GRIFFITHS, John. "Legal pluralism and the theory of legislation-with special reference to the regulation of Euthanasia». En Hanne Petersen y Henrik Zahele (editores). Legal polycentricity: consequences of pluralism in law. Aldershot, Brookfield, Hong Kong y Sydney: Dartmouth, 1995; "Legal pluralism and the social working of law». En Bob Brouwer et al. (editores). Coherence and conflict in law. Proceedings of the $3^{\text {rd }}$ Benelux-Scandinavian symposium in legal theory. Ámsterdam: Kluwer Law and Taxation Publishers Deventer, 1992; «Legal pluralism and the social working in law». En A. Soeteman (editor). Coherence and conflicts concerning the law. Zwolle: Tjeenk Willink, 1991; «What is legal pluralism?». Journal of Legal Pluralism, № 24, 1986, pp. 1-50; «The legal integration of minority groups set in the context of legal pluralism». En University of Leiden. Staatsrecht en Minderherdsgroepen. Leiden: Leiden University Press, 1979.

6 MerRY, Sally Engle. «Legal pluralism». Law \& Society Review, vol. 22, № 5, 1988; Getting justice and getting even: legal consciousness among working-class Americans. Chicago: University of Chicago Press, 1990; The possibility of popular justice: a case study of American community mediation (coeditado con Neal Milner y Ann Arbor). Michigan: University of Michigan Press, 1993; Human rights and gender violence: translating international law into local justice. Chicago: University of Chicago Press, 2006

7 FOBLETS, Marie-Claire. Introduction to the anthropological study of legal systems, Readings Book. Leuven: Uitgeverij Acco, 2002; "South-African woman caught between tradition and the new Constitution: some elements for reflection based on the work of the South African Law Commission". Recht in Afrika Journal, 2001, pp. 105-122; "Cultural depicts: the repercussion of cultural conflicts on delinquent behavior. Reflections on the contribution of legal Anthropology to a contemporary debate". European Journal of Crime, Criminal law and Criminal Justice, vol. 6/3, 1998, pp. 187-207.

8 Weber, Max. Economía y sociedad. México: Fondo de Cultura Económica, 1974 [1922]. 


\section{III.1. Experiencia de la sociología del Derecho en el tra- bajo de investigación}

Si partimos del mismo ejemplo —el del conflicto de violencia física entre los miembros de un matrimonio-, cabe aclarar que el Derecho y la sociología comparten conocimientos, métodos y técnicas diferentes en el trabajo de investigación. El Derecho tiene una aproximación lógica normativa al conflicto, como hicimos notar al referirnos a la experiencia de la antropología del Derecho. En términos puros, el Derecho, o la consideración jurídica, tiene un contenido ideal basado en la norma jurídica, como diría Weber: «[La consideración jurídica] se pregunta por lo que idealmente vale como Derecho. Esto es, qué significa o, lo que es lo mismo, qué sentido normativo lógicamente correcto debe corresponder a una formación verbal que se presenta como norma jurídica $»^{9}$ (subrayado nuestro).

La norma jurídica es la base del conocimiento, método y técnicas del Derecho. El trabajo de investigación tiene que tenerla en cuenta, y su utilización coherente en el ordenamiento jurídico, si es que se quiere hacer un trabajo de Derecho. Omitirla significaría orientar la investigación por otra disciplina ajena a este.

La sociología, en cambio, se pregunta por los hechos y el orden social. De forma semejante a la antropología, estudia las causas de las conductas y relaciones humanas, pero prioriza su dimensión social. Para la sociología, importa más encontrar causas que se puedan generalizar para explicar el conflicto de violencia familiar de la pareja, y no tanto encontrar causas específicas limitadas solo al conflicto o fenómeno de estudio.

Se trata de una explicación sobre la base de probabilidades, como dice el mismo Weber: «[La sociología] se pregunta lo que de hecho ocurre en una comunidad en razón de que existe la probabilidad de que los hombres que participan en la actividad comunitaria, sobre todo aquellos que pueden influir considerablemente en esa actividad, consideren subjetivamente como válido un determinado orden y orienten por él su conducta práctica ${ }^{10}$ (subrayado nuestro).

En el trabajo de investigación, la sociología aporta el estudio de los hechos sobre la base de las probabilidades que se deducen del propio actuar de los hombres y mujeres de una comunidad determinada. La sociología estudia, además, las causas de ese actuar, pero lo hace para proyectar el propio actuar sobre la base de probabilidades. Es decir, convierte las causas en probabilidades. Para ello, esta disciplina recurre al método del trabajo de campo, pero prefiere los cuestionarios y las encuestas más que la observación y las entrevistas; trata de transformar datos cualitativos en cuantitativos. Así, si retomamos el mismo ejemplo, la sociología trata

\section{LA ANTROPO- \\ LOGÍA Y LA \\ sociología deL DERECHO COMO FORMACIÓN \\ INTER-}

DISCIPLINARIA 
de obtener datos cuantitativos que le permitan entender el caso y proyectarlo a otros semejantes.

Al tener en cuenta estas diferencias de conocimientos, métodos y técnicas, es que el Derecho y la sociología se complementan para tener una aproximación objetiva y valorativa en el trabajo de investigación. La aproximación objetiva la otorga la sociología, y la aproximación valorativa la aporta el Derecho. Ambos tratan el fenómeno o caso de investigación explicando la conducta de los hombres y mujeres como hecho y como Derecho. Este último aporta el estudio de la validez de la norma, en tanto la primera aporta la comprensión de la probabilidad de su cumplimiento.

De vuelta al ejemplo, el Derecho y la sociología en su trabajo de investigación interdisciplinario explicarán por qué los miembros del matrimonio incumplen la norma que obliga el respeto mutuo de la pareja. A partir de este caso, y de otros similares, construirán una explicación objetiva que, basada en probabilidades, puede preveer el mismo desenlace en otros casos, o puede elaborar políticas que prevean casos futuros y busquen superar los existentes. La norma como valor y como hecho es asumida por ambas disciplinas.

\section{III.2. La experiencia de la sociología del Derecho en el trabajo educativo}

Si seguimos la misma explicación dada en la experiencia de la antropología jurídica, la interacción e integración del Derecho y la sociología permiten el aprendizaje y enseñanza de un tema o caso más allá de lo que dice la ley o la norma, lo comprende en un contexto de pluralismo jurídico y utiliza para ello el método del trabajo de campo. Sin embargo, la sociología y el Derecho como experiencia interdisciplinaria busca un aprendizaje y enseñanza con mayores resultados, o muestras más generales, que la conecta con el ejercicio de políticas de gobierno o políticas sociales en general. Los datos cuantitativos y el uso de probabilidades se orientan a estos resultados.

Esto significa que la sociología y el Derecho, en su trabajo educativo, permiten formar abogados con una perspectiva más amplia que aquella basada solo en el estudio de la norma, y, por otro lado, permiten formar sociólogos que no se quedan solo en el estudio de los hechos y las probabilidades sin tener en cuenta la preexistencia de un orden normativo lógico y sistemático por alcanzar o mejorar.

De ahí que uno de los aportes de la interacción de la sociología con el Derecho sea la teoría del Derecho como instrumento de cambio social. Es sobre la base de la probabilidad de los hechos que el Derecho se puede utilizar como medio para producir un cambio de valores que responden a las prácticas de un grupo social determinado. Es, además, sobre la base de la misma teoría que se puede entender que toda norma 
transmite valores y que su objetivo final es que esos valores sean comprendidos para que se produzca su internalización en el receptor y, con ello, en el grupo social ${ }^{11}{ }^{12}$.

La labor educativa del Derecho y la sociología se integra justo dentro de esta perspectiva. Si retomamos el ejemplo del conflicto de violencia física entre los miembros de un matrimonio, la práctica educativa de la sociología del Derecho se pregunta por las causas y efectos del tema o problema, pero, sobre todo, piensa en la idea o propuesta de cambios y mejoras en ese conflicto dado. Si bien cualquier propuesta está sujeta a debate, la información objetiva, o cuantitativa, que presenta la sociología del Derecho ayuda a orientar una solución.

Para el aprendizaje y la enseñanza de la sociología del Derecho también destaca una gama de autores especialistas que combinaron ambas disciplinas en sus estudios. Uno de los más reconocidos es Max Weber ${ }^{13}$, como hemos indicado, pero también se suman Renato Treves ${ }^{14}$, George Gurvitch $^{15}$, Roscoe Pound ${ }^{16}$, Boaventura de Sousa Santos ${ }^{17}$ y William Evan $^{18}$, entre otros autores, que se acercan a la comprensión de conflictos o temas como el planteado.

\section{RETOS Y DESAFÍOS EN EL TRABAJO INTERDISCIPLINARIO}

Los temas expuestos nos llevan a plantearnos algunas interrogantes sobre el trabajo interdisciplinario que involucran la antropología y la sociología con el Derecho. Estas son preguntas que cuestionan la situación actual y proyectan algunas perspectivas sobre la interacción e integración de dichas disciplinas. Entre estas preguntas podemos identificar:

- ¿Qué disciplina es más importante cuando se interrelacionan dos o más disciplinas?

11 MASSELL, Gregory. «El Derecho como instrumento de cambio revolucionario en un medio tradicional: el caso del Asia Central Soviética». En Lorenzo Zolezzi (editor). Materiales de enseñanza de Sociología del Derecho. Lima: PUCP, 1977.

12 Evan, William. «El Derecho como instrumento de cambio social». En Lorenzo Zolezzi (editor). Materiales de enseñanza de Sociología del Derecho. Lima: PUCP, 1977.

13 WEBER, Max. Ob. cit.

14 TREVES, Renato. Sociología del Derecho. Barcelona: Ariel, 1988

15 GURVITCH, George. Traité de sociologie. París: Presses Universitaires de France, 1968; Sociology of law. Londres y Boston: Routledge \& Kegan Paul, 1974 [1947].

16 Pound, Roscoe. «Sociology of law». En George Gurvitch y Wilbert E. Moore (editors). Twentieth Century Sociology. Nueva York: The Philosophical Library, 1945; Sociology of law and sociological jurisprudence. Toronto: University of Toronto, 1943; "A theory of social interest». Papers and Proceedings of the American Sociological Society, № 15, Nueva York, 1921.

17 SANTOS, Boaventura de Sousa. Towards a new common sense. Law science and politics in the paradigmatic transition. Nueva York y Londres: Routledge, 1995; "Law and community: the changing nature of State power in late capitalism». En Richard Abel (editor). The politics of informal Justice. Volume II: The American experience. Nueva York: Academic Press, 1982; «The Law of the Oppressed: The Construction and Reproduction of Legality in Pasargada Law». Law and Society Review, $\mathrm{N}^{\circ} 12,1977$, pp. 5-126.

18 Evan, William M. The sociology of law. A social-structural perspective. Nueva York y Londres: The Free Press and Collier Macmillan Publishers, 1980; Social structural and law. Theoretical and empirical perspective. Londres y Nueva Delhi: SAGE Publications, Newbury Park, 1990.

LA ANTROPO-

LOGÍA Y LA

SOCIOLOGÍA DEL DERECHO COMO FORMACIÓN INTERDISCIPLINARIA 
- ¿Qué conflictos éticos se presentan en la ejecución de la actividad de investigación y en el trabajo educativo interdisciplinario?

- ¿Hacía dónde dirigir las nuevas inquietudes del trabajo interdisciplinario?

Sobre la primera pregunta, consideramos que cuando interrelacionamos el Derecho con la antropología o la sociología, ambas disciplinas son importantes. No cabe pensar ni proponer la hegemonía de alguna de ellas. En caso de incompatibilidad del uso del contenido, método o técnicas de las disciplinas respecto del problema que se investiga o que es objeto de práctica educativa, cabe promover el diálogo de estas.

Por ejemplo, frente al caso del conflicto por violencia física entre los miembros de un matrimonio, el método del Derecho buscará priorizar los valores mientras que el método de la antropología o de la sociología buscará priorizar la comprensión de la situación de la pareja. Así, quien maneje el Derecho buscará denunciar el hecho, y quien maneja la antropología o la sociología buscará recopilar más información que explique y acepte la situación. En cualquier caso, lo ideal es buscar la compatibilidad de ambos métodos: sacrificar parte de los intereses metodológicos dentro del propósito de cumplir con la investigación o actividad educativa. No hay un método que tenga prioridad sobre el otro.

El ejemplo propuesto también nos conecta con la segunda pregunta, relacionada con los conflictos éticos. Cuando se tiene el problema de investigación o actividad educativa, como es el caso de violencia física presentado, icómo debe tomarse el estudio de las normas vinculadas al conflicto? ¿Cómo hecho, tal como lo promueven la antropología y la sociología? O, icómo mandato u orden, tal como lo promueve el Derecho?

Si optamos por lo primero tendremos que aceptar la realidad del problema sin alterarlo, más allá de las normas formales que regulan el conflicto, porque también hay que comprender las normas que de hecho comparten las partes del conflicto, entre ellas y con su grupo social. Si optamos por lo segundo, tenemos que someter la realidad encontrada a las normas del Estado y, entonces, el conflicto de pareja que usamos como ejemplo aparece de antemano prejuzgado y absolutamente rechazado, sin importar la existencia de normas propias que involucran a sus actores. Frente a este conflicto ético, nuevamente, es el diálogo de las disciplinas el que hace posible una solución de compatibilidad.

Las dos cuestiones previas muestran lo difícil del trabajo interdisciplinario, pero, al mismo tiempo, destacan lo importante de su uso frente a situaciones complejas. Dadas estas condiciones, la tercera interrogante se presenta como una alternativa, ihacia dónde dirigir el trabajo interdisciplinario? Cabe dirigirlo a nuevos rumbos, donde, justamente, las cuestiones previas puedan ser reflexionadas y aclaradas. Esto significa 
la orientación hacia nuevos temas de investigación y nuevas actividades educativas.

Es solo con cada investigación, como con cada actividad educativa, que se podrá conocer la efectividad y el desarrollo de los métodos y técnicas de cada disciplina para así resolver los problemas de hegemonía y los conflictos éticos que se presenten. El reto consiste en multiplicar y reforzar las actividades de investigación y educación interdisciplinarias. Para ello, es imprescindible apoyar a los jóvenes investigadores, así como a los jóvenes docentes, pero, sobre todo, a los estudiantes del pregrado. Son ellos quienes tienen en sus manos la mejor comprensión de los problemas actuales, porque experimentan cómo afrontarlos en su tratamiento

LOGÍA Y LA

SOCIOLOGÍA deL DERECHO COMO FORMACIÓN interdisciplinario. 